PROCEEDINGS OF THE

AMERICAN MATHEMATICAL SOCIETY

Volume 139, Number 12, December 2011, Pages 4435-4443

S 0002-9939(2011)10831-1

Article electronically published on April 13, 2011

\title{
SOUL THEOREM FOR 4-DIMENSIONAL TOPOLOGICALLY REGULAR OPEN NONNEGATIVELY CURVED ALEXANDROV SPACES
}

\author{
JIAN GE \\ (Communicated by Jianguo Cao)
}

\begin{abstract}
In this paper, we study the topology of topologically regular 4dimensional open nonnegatively curved Alexandrov spaces. These spaces occur naturally as the blow-up limits of compact Riemannian manifolds with lower curvature bound. These manifolds have also been studied by Yamaguchi in his preprint. Our main tools are gradient flows of semi-concave functions and critical point theory for distance functions, which have been used to study the 3-dimensional collapsing theory in the paper by Cao and Ge. The results of this paper will be used in our future studies of collapsing 4-manifolds, which will be discussed elsewhere.
\end{abstract}

\section{INTRODUCTION}

The topology of a noncompact manifold with a complete metric of nonnegative sectional curvatures was studied by Gromoll-Meyer in GM69 and Cheeger-Gromoll in CG72.

Theorem 0.1 (Soul Theorem, [CG72]). Let $M^{n}$ be an n-dimensional noncompact manifold with a complete metric of nonnegative sectional curvature. Then there exists a compact totally geodesic embedded submanifold $S \subset M$ with nonnegative sectional curvature, such that $M^{n}$ is diffeomorphic to the normal bundle $\nu(S)$ of $S$ in $M^{n}$.

If in addition to the assumption above there exists $p \in M^{n}$ such that the sectional curvatures at $p$ are all positive, then $M^{n}$ is diffeomorphic to $\mathbb{R}^{n}$. This is called Cheeger-Gromoll soul conjecture. It was proved by Perelman in Per94.

For Alexandrov spaces (see BGP92] and BBI01] for basics of Alexandrov spaces) Perelman proved a similar result:

Theorem 0.2 (Soul Theorem for Alexandrov space, [Per91]). Let $X^{n}$ be an $n$ dimensional noncompact Alexandrov space with nonnegative curvature. Then there exists a closed totally convex subset $S \subset X^{n}$, such that $S$ is a deformation retraction of $X^{n}$.

Similar to the Soul Theorem, Cao-Dai-Mei CDM07, CDM09 proved that if in addition to the conditions of Theorem 0.2 one assumes that $X^{n}$ has positive curvature in a metric ball, then $X^{n}$ is contractible. Unlike the manifold case,

Received by the editors September 27, 2010 and, in revised form, October 8, 2010.

2010 Mathematics Subject Classification. Primary 53C20.

(C)2011 American Mathematical Society Reverts to public domain 28 years from publication 
Theorem 0.2 is the best topological result one can expect, in the sense that in general $X^{n}$ is not homeomorphic to the normal bundle of $S$.

By Perelman's stability theorem, if Alexandrov space is the limit of a sequence of Riemannian manifolds with lower curvature bound, then it is a topological manifold. In fact Kapovitch showed the following thoerem in Kap02.

Theorem 0.3 (|Kap02]). If $X^{n}$ is the limit of a sequence of $n$-dimensional Riemannian manifolds with the same lower curvature bound $k$, then $\Sigma_{p} X^{n}$ is homeomorphic to the $(n-1)$-sphere $\mathbf{S}^{n-1}$ for any $p \in X^{n}$. Moreover, all the iterated spaces of directions are homeomorphic to spheres.

It is still unknown whether an Alexandrov space, which satisfies the conclusion of Theorem 0.3 can be realized as a limit of Riemannian manifolds with the same dimension and the same lower curvature bound. In this paper, we consider the class of 4-dimensional topologically regular open nonnegatively curved Alexandrov spaces, in the sense that all space of directions are spheres. These Alexandrov spaces occur naturally as the blow-up limits of compact Riemannian manifolds with lower curvature bound, and thus play an important role in the study of collapsing under a lower curvature bound. We will prove the following:

Theorem 0.4 (Main Theorem). Let $X^{4}$ be as above and $S$ be a soul of $X^{4}$. Then $X^{4}$ is homeomorphic to an open disk bundle over $S$ :

(1) If $\operatorname{dim} S=0$, then $X^{4}$ is homeomorphic to $\mathbb{R}^{4}$.

(2) If $\operatorname{dim} S=1$, then $X^{4}$ is homeomorphic to $\mathbb{R}^{3}$ bundle over $\mathbf{S}^{1}$.

(3) If $\operatorname{dim} S=2$, then $X^{4}$ is homeomorphic to $\mathbb{R}^{2}$ bundle over $S$, where $S=$ $\mathbf{S}^{2}, \mathbb{R P}^{2}, \mathbf{T}^{2}$ or $\mathbf{K}^{2}$.

(4) If $\operatorname{dim} S=3$, then $X^{4}$ is homeomorphic to line bundle over $S$, where $S=$ $\mathbf{S}^{3} / \Gamma, \mathbf{T}^{3} / \Gamma,\left(\mathbf{S}^{2} \times \mathbf{S}^{1}\right) / \Gamma$, and $\Gamma$ some subgroup of isometric group of $S$ acting freely on $S$.

This theorem will be used to study the collapsing 4-manifolds, which will be discussed elsewhere. Theorem 0.4 has also been studied in the preprint [Yam02, Chapters 15 and 16, in a traditional way. Our main tools are the gradient flow of semi-concave functions and Perelman's version of the Fibration Theorem, which have been used extensively in CaoG10] to study the 3-dimensional collapsing manifolds under a lower curvature bound.

\section{Construction of SOUL}

In this section we recall Cheeger-Gromoll's construction of soul for $X^{n}$, where $X^{n}$ is an $n$-dimensional nonnegatively curved open complete Alexandrov space (cf. CG72, Per91]).

Fix $p \in X^{n}$; the Busemann function can be defined by

$$
b(x)=\lim _{t \rightarrow \infty}[\mathrm{d}(x, \partial(B(p, t)))-t],
$$

where $B(p, t)$ is the ball centered at $p$ with radius $t$. Denote the super-level set $b^{-1}([a,+\infty))$ by $\Omega^{a}$. We have

Proposition 1.1 ([CG72, Per91, Wu79]). Let $X^{n}, \Omega^{a}$ be as above. Then the following hold:

(1) The Busemann function $b$ is concave and bounded above.

(2) $\Omega^{a}$ is compact and totally convex for all $a \leq a_{0}:=\max _{x \in X^{n}} b(x)$. 
(3) $a<b \leq a_{0}$ implies $\Omega^{b} \subset \Omega^{a}$ and

$$
\Omega^{b}=\left\{x \in \Omega^{a} \mid \mathrm{d}\left(x, \partial \Omega^{a}\right) \leq b-a\right\} .
$$

(4) There is a filtration of $\Omega(0):=\Omega^{a_{0}}$ by totally convex sets:

$$
\Omega(0) \supset \Omega(1) \supset \cdots \supset \Omega(k),
$$

where $\Omega(i+1)$ is the maximum set of $f_{i}(x)=\mathrm{d}_{\Omega(i)}(x, \partial \Omega(i))$, and $\partial \Omega(k)=\varnothing$.

We call $S=\Omega(k)$ a soul of the type $(s, m)$ if the dimension of the soul is $s$ and the dimension of $\Omega(0)$ is $m$.

We call a geodesic $\gamma:(-\infty,+\infty) \rightarrow X^{n}$ a line in a metric space $X^{n}$ if $\mathrm{d}(\gamma(t), \gamma(s))$ $=|t-s|$ for $\forall t, s \in \mathbb{R}$. The splitting theorem reduces our discussion of 4 dimension to 3 dimension when $X^{4}$ admits a line.

Theorem 1.2 (GP89). Let $X^{n}$ be an open nonnegatively curved Alexandrov space and assume that $X^{n}$ admits a line. Then $X^{n}$ splits isometrically as $X^{n}=N^{n-1} \times \mathbb{R}$, where $N^{n-1}$ is an $(n-1)$-dimensional open nonnegatively curved Alexandrov space.

In order to handle the nonsmooth metric Alexandrov space, Perelman's Stability Theorem and his version of the Fibration Theorem are extensively used in this paper. Let us recall these results.

Theorem 1.3 (Stability Theorem [Per91, Kap07). Let $\left\{X_{\alpha}^{n}\right\}_{\alpha=1}^{\infty}$ be a sequence of $n$-dimensional Alexandrov spaces with curv $\geq-1$ converging to an Alexandrov space with the same dimension: $\lim _{\alpha \rightarrow \infty} X_{\alpha}^{n}=X^{n}$. Then $X_{\alpha}^{n}$ is homeomorphic to $X^{n}$ for $\alpha$ large.

The Stability Theorem for pointed spaces can be stated in a similar way. The Fibration Theorem states that

Theorem 1.4 (Fibration Theorem Per91, Per93). Let $X^{n}$ be an $n$-dimensional Alexandrov space, $U$ a domain in $X^{n}$, and $f: U \rightarrow \mathbb{R}^{k}$ be an admissible function having no critical point and proper on $U$. Then its restriction to this domain is a locally trivial fiber bundle.

We refer to [Per91] for the definitions of admissible functions and a regular map.

\section{Soul Theorem for a 3-Dimensional Alexandrov space}

The topology of 3-dimensional open nonnegatively curved Alexandrov space was studied in SY00] (cf. also CaoG10).

Theorem 2.1 ([SY00]). Let $X^{3}$ be an open complete 3 -manifold with a possibly singular metric of nonnegative curvature. Suppose that $X^{3}$ is oriented and $S^{s}$ is a soul of $X^{3}$. Then the following are true:

(1) When $\operatorname{dim}\left(S^{s}\right)=1$, the soul of $X^{3}$ is isometric to a circle. Moreover, its universal cover $\tilde{X}^{3}$ is isometric to $\tilde{X}^{2} \times \mathbb{R}$, where $\tilde{X}^{2}$ is homeomorphic to $\mathbb{R}^{2}$.

(2) When $\operatorname{dim}\left(S^{s}\right)=2$, the soul of $X^{3}$ is homeomorphic to $\mathbf{S}^{2} / \Gamma$ or $\mathbf{T}^{2} / \Gamma$. Furthermore, $X^{3}$ is isometric to one of four spaces: $\mathbf{S}^{2} \times \mathbb{R}, \mathbb{R P}^{2} \ltimes \mathbb{R}=\left(\mathbf{S}^{2} \times \mathbb{R}\right) / \mathbb{Z}_{2}$, $\mathbf{T}^{2} \times \mathbb{R}$ or $\mathbf{K}^{2} \ltimes \mathbb{R}=\left(\mathbf{T}^{2} \times \mathbb{R}\right) / \mathbb{Z}_{2}$ and $\mathbb{R P}^{2} \ltimes \mathbb{R}$, which is homeomorphic to $\left[\mathbb{R P}^{3}-D^{3}\right]$.

(3) When $\operatorname{dim}\left(S^{s}\right)=0$, the soul of $X^{3}$ is a single point and $X^{3}$ is homeomorphic to $\mathbb{R}^{3}$.

Throughout this paper $\mathbf{S}^{n}$ denotes the standard $n$-sphere, $\mathbf{T}^{n}$ denotes the $n$ dimensional torus, $\mathbf{K}^{2}$ denotes the Klein bottle and $D^{3}$ denotes the standard 3-ball. 


\section{Proof of the Main Theorem}

A key observation by K. Grove is that the distance function to the soul has no critical point in $X^{n}-S^{s}$ when $X^{n}$ is a smooth Riemannian manifold. However this is no longer true for Alexandrov spaces, even for a topologically regular one. For example, let $L$ be the closed half strip $\left\{(x, y) \in \mathbb{R}^{2} \mid x \geq 0,0 \leq y \leq 1\right\}$. Then the double of $L$ which is denoted by $\operatorname{dbl}(L)$ is a 2-dimensional Alexandrov space with nonnegative curvature, which is homeomorphic to $\mathbb{R}^{2}$ and has point soul $\left(0, \frac{1}{2}\right)$. It is clear that the distance function has critical points $(0,0)$ and $(0,1)$. However for Alexandrov space, CaoG10 derived a modified result similar to Grove's observation.

Proposition 3.1 ([CaoG10, Proposition 2.5). The function $f(x)=\mathrm{d}_{X^{n}}(x, A)$ has no critical point for $x \in\left[X^{n}-\Omega(0)\right]$, where $A \subset \Omega(0)$.

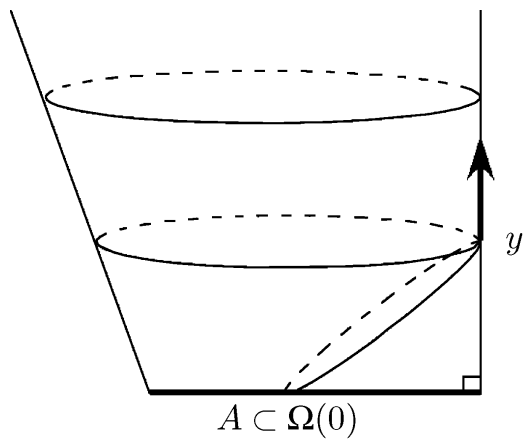

FigURE 1. $\mathrm{d}_{X^{n}}(x, A)$ has no critical point in $\left[X^{n}-\Omega(0)\right]$

For simplicity we assume that $a_{0}=0$ by adding a constant if needed. Let $A=$ $\Omega(0)$. Using Proposition 3.1, we see that $f(x)=\mathrm{d}(x, A)$ has no critical value within $(\varepsilon,+\infty)$. It follows by Perelman's Fibration Theorem that $X^{n} \cong B_{X^{n}}(\Omega(0), \varepsilon)$ for $\varepsilon>0$, where $B_{X}(A, r)$ denotes the set of points with distance $\leq r$ to a set $A$ in the metric $X$.

We will divide the proof of our Main Theorem into the following cases.

3.1. Soul of the type $(s=3, m=3), X^{4}=S \times \mathbb{R}^{1}$ or $\left(S \times \mathbb{R}^{1}\right) / \mathbb{Z}_{2}$.

Proof (cf. the proof of Theorem 2.21 in CaoG10). In this case, $S=\Omega(0)$ and has dimension 3. Since $X^{4}$ is topologically regular, $S$ is a topological manifold and nonnegatively curved. Hence by Hamilton's classification of 3-dimensional manifolds with nonnegatively Ricci curvature, $S$ is homeomorphic to $\mathbf{S}^{3} / \Gamma, \mathbf{T}^{3} / \Gamma$ or $\mathbf{S}^{2} \times \mathbf{S}^{1} / \Gamma$, where $\Gamma$ is a subgroup of the isometry group of $\mathbf{S}^{3}, \mathbf{T}^{3}, \mathbf{S}^{2} \times \mathbf{S}^{1}$.

For $p \in S$, we know $\Sigma_{p}(S)$ is homeomorphic to $\mathbf{S}^{2}$, which divides $\Sigma_{p}\left(X^{4}\right) \cong \mathbf{S}^{3}$ into two parts, denoted by $A^{ \pm}$. Since $S$ is totally convex, $\Sigma_{p}(S)$ is convex in $\Sigma_{p}\left(X^{4}\right)$; therefore $\left.r_{\Sigma_{p}(S)}\right|_{A^{ \pm}}$has a unique maximum point $\xi^{ \pm}$in $A^{ \pm}$. Denote the maximum values by $\ell^{ \pm}$, i.e. $r_{\Sigma_{p}(S)}\left(\xi^{ \pm}\right)=\ell^{ \pm}$. Since $\Omega(0)=S$ is the set of maximum points for the Busemann function, by the first variation theorem we know $\ell^{ \pm} \geq \pi / 2$. On the other hand if $\gamma:[0, \ell] \rightarrow \Sigma_{p}\left(X^{4}\right)$ is a shortest geodesic connecting $\xi^{-}$to $\xi^{+}$, 
then let $t_{0} \in[0, \ell]$ satisfy $\gamma\left(t_{0}\right) \in \Sigma_{p}(S)$. By triangle inequality we know that

$$
\begin{aligned}
\mathrm{d}\left(\xi^{-}, \xi^{+}\right) & =\mathrm{d}\left(\xi^{-}, \gamma\left(t_{0}\right)\right)+\mathrm{d}\left(\gamma\left(t_{0}\right), \xi^{+}\right) \\
& \geq \mathrm{d}\left(\Sigma_{p}(S), \xi^{-}\right)+\mathrm{d}\left(\Sigma_{p}(S), \xi^{+}\right) \\
& =\ell^{-}+\ell^{+} \\
& \geq \frac{\pi}{2}+\frac{\pi}{2} \\
& =\pi .
\end{aligned}
$$

Note that $\operatorname{curv}\left(\Sigma_{p}\left(X^{4}\right)\right) \geq 1$ implies $\operatorname{diam}\left(\Sigma_{p}\left(X^{4}\right)\right) \leq \pi$. Hence the inequalities in (3.1) are equalities. In particular $\ell^{-}=\ell^{+}=\pi / 2$ and $\Sigma_{p}\left(X^{4}\right)$ is the spherical suspension over $\Sigma_{p}(S)$; i.e. $T_{p}\left(X^{4}\right)$ splits isometrically as $T_{p}(S) \times \mathbb{R}^{1}$. Hence we have a normal line bundle over $S$. By passing to the double cover, we can assume that this line bundle is trivial. Therefore $S$ separates $X^{4}$ into two parts and $X^{4}$ has two ends. Now it is easy to see $X^{4}$ admits a line. By the Splitting Theorem, $X^{4}$ is isometric to $S \times \mathbb{R}^{1}$.

3.2. Soul of the types $(s=0$ or $2, m=3), X^{4} \cong \mathbb{R}^{4}$ or $\mathbb{R}^{2} \hookrightarrow X^{4} \rightarrow \Sigma^{2}$, where $\Sigma^{2} \cong \mathbf{S}^{2} / \Gamma, \mathbf{T}^{2} / \Gamma$.

Proof. In this case, $\Omega(0) \cong D^{3}$ or an $I$-bundle over $S \cong \mathbf{S}^{2}, \mathbb{R} \mathbb{P}^{2}, \mathbf{T}^{2}$ or $\mathbf{K}^{2}$ by Theorem 2.1. By the proof of 3.1, the interior of $\Omega(0)$ admits a normal line bundle. Thus, we only have to show that $B_{X^{4}}(\Omega(0), \varepsilon) \cong B_{X^{4}}\left(\Omega^{-\delta}(0), \varepsilon\right)$ for $0<\varepsilon \ll \delta$, where $\Omega^{-\delta}(0):=\{x \in \Omega \mid \mathrm{d}(x, \partial \Omega(0)) \geq \delta\}$.

By Proposition 3.1, $r_{\partial \Omega(0)}(x)=\mathrm{d}(\partial \Omega(0), x)$ has no critical point for $x \in X^{4} \backslash$ $\Omega(0)$. When we restrict to $\Omega(0), r_{\partial \Omega(0)}$ is concave; hence it has no critical value in $(0, a)$ for $a$ small enough. Combining these two facts, we know that there exists $\delta>0$ such that $r_{\partial \Omega(0)}$ has no critical point in $B_{X^{4}}(\partial \Omega(0), 100 \delta)$.

By the lower semi-continuity of the norm of the gradient of the $\lambda$-concave function $\nabla r_{\partial \Omega(0)}$ (cf. Petr07. Corollary 1.3.5), $r_{\partial \Omega(0)}$ has no critical point in $B_{X^{4}}\left(\Omega(0) \backslash \Omega^{-2 \delta}(0), \varepsilon\right)-\partial \Omega(0)$ for $\varepsilon \ll \delta$ small enough. Therefore $r_{\partial \Omega(0)}$ is regular in $B_{X^{4}}\left(\Omega^{-\varepsilon}(0) \backslash \Omega^{-2 \delta}(0), \varepsilon\right)$. By the Fibration Theorem we have

$$
B_{X^{4}}(\partial \Omega(0), \varepsilon) \cong B_{X^{4}}\left(\Omega(0) \backslash \Omega^{-2 \delta}(0), \varepsilon\right)
$$

for $\varepsilon \ll \delta$; see Figure 2 ,



Figure 2. Bundle structure around $\partial \Omega(0)$ 
By the proof of 33.3 we know $B_{X^{4}}(\partial \Omega(0), \varepsilon)$ is homeomorphic to a $D^{2}$ bundle over $\partial \Omega(0)$ :

$$
D^{2} \hookrightarrow B_{X^{4}}(\partial \Omega(0), \varepsilon) \rightarrow \partial \Omega(0)
$$

since $\partial \Omega^{-\varepsilon / 2}(0) \cong \partial \Omega(0)$ and there is a normal line bundle over $\partial \Omega^{-\varepsilon / 2}(0)$. By passing to the double cover one can assume this line bundle is trivial; let $\Gamma$ be the $(\varepsilon / 100)$-section of this line bundle. Clearly $\Gamma \subset B_{X^{4}}(\partial \Omega(0), \varepsilon)$, which implies that the bundle (3.3) admits a global nowhere vanishing section, hence it is a trivial $D^{2}$ bundle. By the homeomorphism (3.2), we have a trivial $D^{2}$ bundle

$$
B_{X^{4}}\left(\Omega(0) \backslash \Omega^{-2 \delta}(0), \varepsilon\right) \cong \partial \Omega(0) \times D^{2} .
$$

Now consider the function $r_{\Omega^{-10 \delta}}(x)$, which has no critical point in $B_{X^{4}}(\Omega(0) \backslash$ $\left.\Omega^{-9 \delta}(0), \varepsilon\right)-\partial \Omega(0)$. Hence we have a gradient flow of $r_{\Omega^{-10 \delta}(0)}$ on

$$
\begin{aligned}
B_{X^{4}}\left(\Omega^{-\delta}(0) \backslash \Omega^{-2 \delta}(0), \varepsilon\right) & \cong(-\varepsilon,+\varepsilon) \times \partial \Omega(0) \times(\delta, 2 \delta) \\
& \cong \partial \Omega(0) \times D^{2}
\end{aligned}
$$

for $\varepsilon \ll \delta$ small enough, where the homeomorphism follows from the fact that $r_{\partial \Omega(0)}$ is concave in the interior of $\Omega(0)$ and that there is a normal line bundle over the interior of $\Omega(0)$; see $\$ 3.1$.

Since the bundle (3.4) and its sub-bundle (3.5) are both trivial bundles, one can extend the gradient flow of $r_{\Omega^{-10 \delta}(0)}$ on $B_{X^{4}}\left(\Omega^{-\delta}(0) \backslash \Omega^{-2 \delta}(0), \varepsilon\right)$ to a gradient-like flow on $B_{X^{4}}\left(\Omega(0) \backslash \Omega^{-2 \delta}(0), \varepsilon\right)$, which gives the homeomorphism (see Figure 3)

$$
B_{X^{4}}(\Omega(0), \varepsilon) \cong B_{X^{4}}\left(\Omega^{-2 \delta}(0), \varepsilon\right) \text {. }
$$

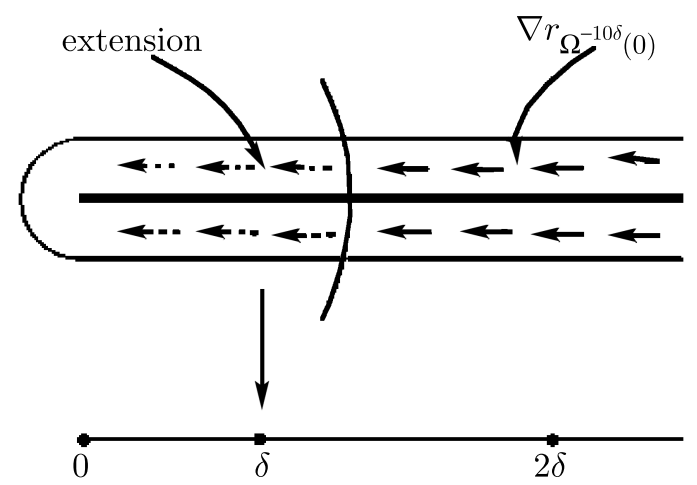

Figure 3. Gradient-like flow on $B_{X^{4}}(\partial \Omega(0), \varepsilon)$

It follows from the proof of $\oint 3.1$ that $B_{X^{4}}\left(\Omega^{-2 \delta}(0), \varepsilon\right)$ is homeomorphic to the normal line bundle over $\Omega^{-2 \delta}(0)$. Hence combined with Theorem 2.1, our Main Theorem holds in these two cases.

3.3. Soul of the type $(s=2, m=2), \mathbb{R}^{2} \hookrightarrow X^{4} \rightarrow S, S=\mathbf{S}^{2} / \Gamma, \mathbf{T}^{2} / \Gamma$.

Proof. In this case, $S$ is a 2-dimensional surface with nonnegative curvature. Thus $S$ is homeomorphic to $\mathbf{S}^{2}, \mathbf{T}^{2}, \mathbb{R P}^{2}$ or $\mathbf{K}^{2}$, by the Splitting Theorem. If $S=\mathbf{T}^{2}$ or $S=\mathbf{K}^{2}$, the universal cover $\widetilde{X^{4}}$ splits isometrically as $N^{2} \times \mathbb{R}^{2}$. Thus the theorem 
follows from the fact that $N^{2}$ is homeomorphic to $\mathbb{R}^{2}$. Now we consider the cases where $S=\mathbf{S}^{2}$ or $\mathbb{R P}^{2}$.

Let $\left\{p_{i}\right\}_{i=1}^{N}$ be the set of extremal points on $S$, where the extremal points in the Alexandrov surface are defined to be the points satisfying $\operatorname{diam}\left(\Sigma_{p_{i}}(S)\right) \leq \pi / 2$. Note that $\Sigma_{p_{i}}(S)=\mathbf{S}^{1}$ is a convex subset of $\Sigma_{p_{i}}\left(X^{4}\right)=\mathbf{S}^{3}$. Hence for $\varepsilon>0$, we have $B_{T_{p_{i}}\left(X^{4}\right)}\left(T_{p_{i}}(S), \varepsilon\right)=T_{p_{i}}(S) \times D^{2}$. Then by the Stability Theorem, we know there exists $\delta>0$ such that $B_{X^{4}}\left(p_{i}, \delta\right) \cap B_{X^{4}}(S, \varepsilon) \cong D^{4}$, and for $\varepsilon \ll \delta$, we have the disc bundle

$$
D^{2} \hookrightarrow B_{X^{4}}\left(p_{i}, \delta\right) \cap B_{X^{4}}(S, \varepsilon) \stackrel{\pi_{i}}{\longrightarrow} B_{S}\left(p_{i}, \delta\right),
$$

where $\pi_{i}$ is the bundle projection map. In particular $\pi_{i}^{-1}\left(\partial B_{S}\left(p_{i}, \delta\right)\right)=D^{2} \times S^{1}$.

For $p \in S \backslash\left(\bigcup_{i=1}^{N} B_{S}\left(p_{i}, \delta / 10\right)\right)$, by our assumption $\Sigma_{p}(S)>\pi / 2$. Thus there exists $\delta^{\prime}>0$ and an admissible map $F_{p}=\left(f_{1}, f_{2}\right): B_{S}\left(p, \delta^{\prime}\right) \rightarrow \mathbb{R}^{2}$ which is regular $B_{S}\left(p, \delta^{\prime}\right)$. By the lower semi-continuity of gradient of semi-concave functions, we know $F_{p}$ is regular in $B_{X^{4}}\left(p, \delta^{\prime \prime}\right)$, for some $\delta^{\prime \prime}>0$ satisfying $\delta^{\prime \prime} \leq \delta \ll \varepsilon$. Thus we have a fiber bundle

$$
N \hookrightarrow B_{X^{4}}\left(p, \delta^{\prime \prime}\right) \stackrel{\pi}{\rightarrow} D^{2} \cong B_{S}\left(p, \delta^{\prime \prime}\right) .
$$

Let $f(x)=\mathrm{d}(x, S)$. Since $G_{p}=\left(f_{1}, f_{2}, f\right)$ is regular in the domain

$$
A_{X^{4}}(S, \varepsilon / 100, \varepsilon) \cap B_{X^{4}}\left(p, \delta^{\prime \prime}\right), \text { for } \varepsilon \ll \delta,
$$

where $A_{X}\left(S, \varepsilon_{1}, \varepsilon_{2}\right)$ denotes the annular region, i.e. all points have distance to $S$ between $\varepsilon_{1}$ and $\varepsilon_{2}$, it follows from the Fibration Theorem that we have a fiber bundle

$$
\mathbf{S}^{1} \hookrightarrow A_{X^{4}}(S, \varepsilon / 100, \varepsilon) \cap B_{X^{4}}\left(p, \delta^{\prime \prime}\right) \rightarrow D^{2} \times I
$$

where $I$ is an open interval. Thus $\partial N=\mathbf{S}^{1}$, and by the generalized Margulis Lemma (cf. FY92]), $N \cong D^{2}$.

Now we can glue the $D^{2}$ bundle together (this part is similar to Yamaguchi's construction in [Yam02, page 102). Let $S\left(\frac{\delta}{2}\right)=S-\bigcup_{i=1}^{N} B\left(p_{i}, \frac{\delta}{2}\right)$. By construction we have a $D^{2}$ bundle over $S\left(\frac{\delta}{2}\right)$ :

$$
D^{2} \hookrightarrow B_{X^{4}}\left(S\left(\frac{\delta}{2}\right), \varepsilon\right) \stackrel{\pi}{\rightarrow} S\left(\frac{\delta}{2}\right) .
$$

Hence $\pi^{-1}\left(\partial B_{S}\left(p_{i}, \frac{\delta}{2}\right)\right)=D^{2} \times S^{1}$. Consider the gradient flow of $r_{p_{i}}(\cdot)=\mathrm{d}\left(p_{i}, \cdot\right)$ on $A_{S}\left(p_{i}, \delta / 2, \delta\right)$. Again by the lower semi-continuity of $\left|\nabla r_{p_{i}}\right|$, for $\varepsilon \ll \delta$ small enough, $r_{p_{i}}$ is regular in $B_{X^{4}}\left(A_{S}\left(p_{i}, \delta / 2, \delta\right), \varepsilon\right)$ and hence provides a homeomorphism $\phi$ between $F_{i}:=\pi^{-1}\left(\partial B_{S}\left(p_{i}, \frac{\delta}{2}\right)\right)$ and $G_{i}:=\pi^{-1}\left(\partial B_{S}\left(p_{i}, \delta\right)\right)$. Clearly $\partial B_{S}\left(p_{i}, \delta / 2\right)$ is isotopic to $\partial B_{S}\left(p_{i}, \delta\right)$ in $B_{S}\left(p_{i}, 2 \delta\right)$, and hence when restricted to the boundaries $\partial F_{i}=\mathbf{S}^{1} \times \mathbf{S}^{1}$ and $\partial G_{i}=\mathbf{S}^{1} \times \mathbf{S}^{1}, \phi$ is isotopic to the identity. Therefore we can glue the $D^{2}$ bundle together.

3.4. Soul of the types $(s=1, m=1,2,3), \mathbb{R}^{3} \hookrightarrow X^{4} \rightarrow \mathbf{S}^{1}$.

Proof. If the soul is $\mathbf{S}^{1}$, then the universal cover $\widetilde{X^{4}}$ admits a line by the total convexity of soul. Therefore, $\widetilde{X^{4}}=N^{3} \times \mathbb{R}$, where $N^{3}$ is homeomorphic to $\mathbb{R}^{3}$ by Theorem 2.1. Thus Theorem 0.4 holds in this case. 


\subsection{Soul of the type $(s=0, m=2), X^{4} \cong \mathbb{R}^{4}$.}

Proof. Since the normal bundle over point soul $S$ is homeomorphic to $\mathbb{R}^{4}$, it is enough to show that $B_{X^{4}}(\Omega(0), \varepsilon) \cong D^{4}$. It is clear that $\Omega(0) \cong D^{2}$ since the soul is a point. By the proof of 3.3 and the fact that $D^{2}$ is contractible, we see that all $D^{2}$ bundles over $\Omega(0) \cong D^{2}$ are trivial, and we have

$$
B_{X^{4}}\left(\Omega^{-100 \varepsilon}, \varepsilon\right) \cong D^{2} \times D^{2} \cong D^{4} .
$$

We claim that $B_{X^{4}}(\partial \Omega(0), \varepsilon) \cong \mathbf{S}^{1} \times D^{3}$. Assume the claim first. By the proof of \$3.2. the gradient of $\mathrm{d}_{X^{4}}\left(\Omega^{-10 \delta}(0), \cdot\right)$ can be extended to $B_{X^{4}}(\partial \Omega(0), \varepsilon)$ and will give the homeomorphism from $B_{X^{4}}\left(\Omega^{-100 \varepsilon}, \varepsilon\right) \cong D^{4}$ to $B_{X^{4}}(\Omega(0), \varepsilon)$.

Proof of the claim. Let $\left\{p_{i}\right\}_{i=1}^{N}$ be the set of extremal points in $\partial \Omega(0)$. By the Stability Theorem, $B_{X^{4}}\left(p_{i}, \varepsilon\right) \cong D^{4}$. Let $\gamma_{i}$ be the boundary curve connection $p_{i}$ to $p_{i+1}$ with the understanding that $p_{N+1}=p_{1}$. One can assume $\gamma_{i}$ is short enough such that $\mathrm{d}_{p_{i}}$ has no critical point in $B_{X^{4}}\left(\gamma_{i}^{-\delta}, \varepsilon\right)$, where $\delta \gg \varepsilon$, and $\gamma_{i}^{-\delta}$ is the sub-curve of $\gamma_{i}$ defined by $\left\{x \in \gamma_{i} \mid \mathrm{d}\left(x, p_{i}\right) \geq \delta\right.$ and $\left.\mathrm{d}\left(x, p_{i+1}\right) \geq \delta\right\}$. Hence by the Fibration Theorem it is a locally trivial fiber bundle,

$$
N^{3} \hookrightarrow B_{X^{4}}\left(\gamma_{i}, \varepsilon\right) \rightarrow \gamma_{i}^{-\delta} .
$$

Since $B_{X^{4}}\left(p_{i}, \varepsilon\right) \cong D^{4}$, we have $S_{X^{4}}\left(p_{i}, \delta\right) \cap B_{X^{4}}\left(\gamma_{i}^{-\delta}, \varepsilon\right) \cong D^{3}$; hence $N^{3} \cong D^{3}$. This completes the proof.

3.6. Soul of the type $(s=0, m=1), X^{4} \cong \mathbb{R}^{4}$.

Proof. The proof is identically the same as Subcase 3.1 of Theorem 2.21 in CaoG10, so we omit it here.

\subsection{Soul of the type $(s=0, m=0), X^{4} \cong \mathbb{R}^{4}$.}

Proof. Let $p=S$ be a soul and $\left\{\alpha_{n}\right\}_{n=1}^{\infty}$ be a sequence of numbers such that $\lim _{n \rightarrow \infty} \alpha_{n}=\infty$. It is clear that $\lim _{n \rightarrow \infty}\left(\alpha_{n} X^{4}, p\right)=\left(T_{p}\left(X^{4}\right), O\right)$. Then it follows by the Stability Theorem that $B_{\alpha_{n} X^{4}}(p, \varepsilon) \cong B_{T_{p} X^{4}}(O, \varepsilon) \cong \mathbb{R}^{4}$. Since $\mathrm{d}_{p}(x)$ has no critical point in $X^{4} \backslash p$, we conclude that $X^{4} \cong \mathbb{R}^{4}$ by Perelman's Fibration Theorem.

This completes the proof of the Main Theorem.

\section{ACKNOWLEDGEMENTS}

The author is indebted to his advisor, Professor Jianguo Cao, for his guidance and support. The author is also grateful to Professor Karsten Grove for many useful discussions.

\section{REFERENCES}

[BBI01] Dima Burago, Yuri Burago, S. Ivanov, A course in metric geometry. Graduate Studies in Mathematics, 33. American Mathematical Society, Providence, RI, 2001. xiv+415 pp. MR1835418 (2002e:53053)

[BGP92] Yu Burago, M. Gromov, G. Perelman, A. D. Aleksandrov spaces with curvatures bounded below. (Russian) Uspekhi Mat. Nauk 47 (1992), no. 2(284), 3-51, 222; translation in Russian Math. Surveys 47 (1992), no. 2, 1-58. MR.1185284 (93m:53035)

[CDM07] Jianguo Cao, Bo Dai, Jiaqiang Mei, An extension of Perelman's soul theorem for singular spaces, arXiv:0706.0565v5 [math.DG]. 
[CDM09] J. Cao, Bo Dai, Jiaqiang Mei, An optimal extension of Perelman's comparison theorem for quadrangles and its applications. Advanced Lectures in Mathematics, volume 11, pp. 39-59. In "Recent Advances in Geometric Analysis", edited by Y. Lee, C.-S. Lin, and M.-P. Tsui. Higher Educational Press and International Press, 2009. MR 2648938

[CaoG10] Jianguo Cao, Jian Ge, A simple proof of Perelman's Collapsing Theorem for 3manifolds. The Journal of Geometric Analysis, published online August 5, 2010, DOI 10.1007/s12220-010-9169-5.

[CG72] J. Cheeger, D. Gromoll, On the structure of complete manifolds of nonnegative curvature, Ann. of Math. (2) 96 (1972), 413-443. MR0309010 (46:8121)

[FY92] K. Fukaya, T. Yamaguchi, The fundamental groups of almost nonnegatively curved manifolds, Ann. of Math. (2) 136 (1992), 253-333. MR1185120 (93h:53041)

[GP89] K. Grove, P. Petersen, On the excess of metric spaces and manifolds, preprint.

[GM69] D. Gromoll, W. Meyer, On complete open manifolds of positive curvature, Ann. of Math. (2) 90 (1969), 75-90. MR0247590 (40:854)

[Kap02] V. Kapovitch, Regularity of limits of noncollapsing sequences of manifolds, Geom. Funct. Anal. 12 (2002), no. 1, 121-137. MR1904560 (2003b:53043)

[Kap07] V. Kapovitch, Perelman's Stability Theorem, in "Surveys in differential geometry", Vol. XI. Metric and comparison geometry. Edited by Jeff Cheeger and Karsten Grove. International Press, Somerville, MA, 2007, pp. 103-136. MR2408265 (2009g:53057)

[Per91] G. Perelman, A. D. Alexandrov's spaces with curvatures bounded from below. II, preprint.

[Per93] G. Perelman, Elements of Morse theory on Aleksandrov spaces (Russian summary), Algebra i Analiz 5 (1993), no. 1, 232-241; translation in St. Petersburg Math. J. 5 (1994), no. 1, 205-213. MR.1220498 (94h:53054)

[Per94] G. Perelman, Proof of the soul conjecture of Cheeger and Gromoll, J. Differential Geom. 40 (1994), 209-212. MR1285534 (95d:53037)

[Petr07] A. Petrunin, Semiconcave functions in Alexandrov's geometry. Surveys in differential geometry. Vol. XI. Metric and comparison geometry. Edited by Jeff Cheeger and Karsten Grove. Surveys in Differential Geometry, volume 11. International Press, Somerville, MA, 2007, pp. 137-201. MR2408266 (2010a:53052)

[SY00] T. Shioya, T. Yamaguchi, Collapsing three-manifolds under a lower curvature bound, J. Differential Geom. 56 (2000), 1-66. MR1863020 (2002k:53074)

[Wu79] $\mathrm{H}$. Wu, An elementary method in the study of nonnegative curvature, Acta Math. 142 (1979), no. 1-2, 57-78. MR512212 (80c:53054)

[Yam02] T. Yamaguchi, Collapsing 4-manifolds under a lower curvature bound, 2002 preprint.

Department of Mathematics, University of Notre Dame, Notre Dame, Indiana 46556

E-mail address: jge@nd.edu 